\title{
Research on Crop Image Recognition Technology - Take Daylily as an Example
}

\author{
Jichao Zhao, Fengzhi Dai \\ College of Electronic Information and Automation, Tianjin University of Science and Technology, \\ Tianjin, China, \\ ChinaE-mail:*daifz@tust.edu.cn \\ www.tust.edu.cn
}

\begin{abstract}
Digital image recognition technology is the core technology of agricultural robots. The key research content is to judge the maturity of crops through image recognition. In this paper, the daylily is used as the crop to be identified, and digital image processing technology is used to separate the daylily from the background image, and then send the processed information to the picking actuator of the robot to assist the actuator in picking tasks. This method can not only be used in the recognition of daylily but also can be used in the recognition of tomatoes, cucumbers, and other crops by adjusting the recognition pictures and recognition parameters. It has high scalability.
\end{abstract}

Keywords: Crop Picking; Daylily; Digital Image Recognition; Maturity

\section{Introduction}

Agricultural robot refers to a kind of flexible automation or semi-automation equipment that takes agricultural products as the operating object, and has some human information perception and limb action functions. It is a kind of flexible automation or semi-automation equipment that integrates a variety of disciplines ${ }^{1}$. Agricultural robots can gradually replace human labor and continuously help agricultural production to reduce labor intensity. At the same time, they can also improve labor efficiency and help solve the problem of labor scarcity faced by many countries. Agricultural machinery has been paid more and more attention by developed and developing countries with less agricultural population, and has become one of the focal points of international agricultural equipment industry technology competition.

Agricultural machinery picking operations, first of all through the visual system for the identification and positioning of agricultural products ${ }^{2}$. The performance of the visual system determines the accuracy and efficiency of identification and positioning of agricultural products, and has a direct impact on the picking effect of the robot.

The visual system is generally composed of camera, image processing device, distance measuring device and computer. At present, the number of commonly used cameras is 1 or 2 , namely monocular or binocular vision system. Images of the produce are captured by a camera before picking, then processed to identify the produce and its branches and leaves, and finally to determine the exact spatial coordinates of the produce ${ }^{3-5}$. Finally, actuators such as manipulator are driven to pick agricultural products at a certain Angle and strength. In order to avoid the influence of natural light, artificial light sources or optical filters are often needed to reduce the influence of surface shadows on the recognition effect of agricultural products.

In this paper, a crop recognition algorithm is proposed, which can extract the crops to be recognized from the crop image collected from the camera of agricultural robot. The extraction effect of the algorithm was tested

(C) The 2021 International Conference on Artificial Life and Robotics (ICAROB2021), January 21 to 24, 2021 
with daylily as the main example. The generality of the algorithm is verified by testing on tomato.

\section{Recognition Algorithm}

According to their growth characteristics, crops can be divided into two categories: color changes before and after maturity, and color does not change. For instance, daylily and tomato fall into the category of color change, while cucumbers and beans fall into the category of color un-change.

Daylily is mainly distinguished from the color of its flower, which is cyan when immature and light yellow when mature. As shown in Fig.1, ripe daylilies are on the left and immature daylilies are on the right.
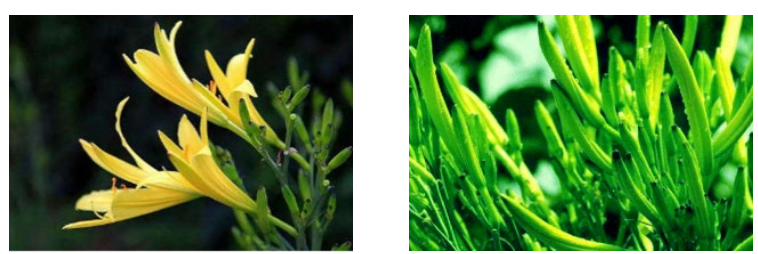

Fig.1. Daylily, left is ripe and right is immature

Our algorithm is mainly applicable to the extraction of crops with different colors before and after maturity. The algorithm flow chart is shown in Fig.2.

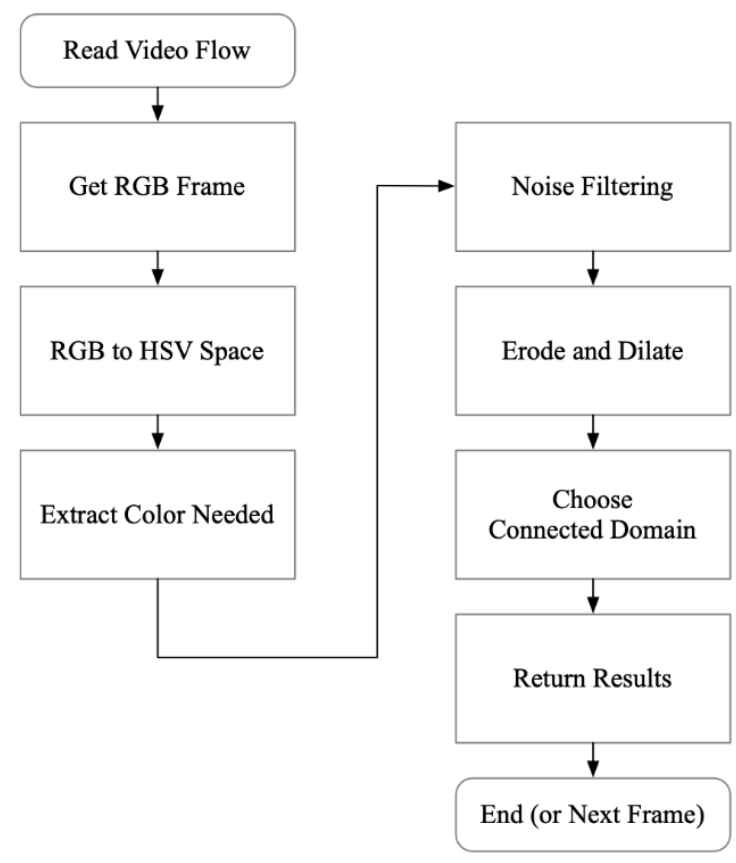

Fig.2. Algorithm Flow

\subsection{HSV Color Space}

The commonly used color space is RGB color space. Each pixel of the image contains three channels consist of Red, Green, Blue, and the final pixel color is composed of three colors mixed together. RGB color space is the cube model. HSV is a kind of color space created based on the intuitive characteristics of colors. It is also known as a Hexcone Model. The RGB color model is hardwareoriented, while the HSV color model is user-oriented. RGB space model and HSV space model are shown in the Fig.3.


Fig.3. Color-Space, left is RGB-Space, right is HSV-Space

In HSV space, $\mathrm{H}$ represents Hue, which is measured at an Angle ranging from $0^{\circ}$ to $360^{\circ}$. The value is calculated counterclockwise from red, which is $0^{\circ}, 120^{\circ}$ in green, and $240^{\circ}$ in blue. Their complementary colors are: yellow is $60^{\circ}$, cyan is $180^{\circ}$ and purple is $300^{\circ}$. S is the Saturation, which indicates the degree of Saturation of colors. If the white component of spectral color is 0 , the saturation value is highest, the color is deep and bright. Generally, the value range of $\mathrm{S}$ is $0 \% \sim 100 \%$. The larger the value, the more saturated the color is. V is Value, representing the brightness of the color, ranging from 0 to 1 .

Compared with RGB space, HSV space can intuitively express the brightness, tone and brightness of colors, which is convenient for color contrast and emotional transmission. Therefore, HSV color space is selected to identify daylily. In addition, the main parameter that affects the identification is Hue, and we constantly adjust the $\mathrm{H}$ value to identify the daylily with good maturity. The effect of daylily extracted through HSV color space is shown in Fig.5(a).

\subsection{Noise Filtering}

When crops are extracted by color, similar color areas that do not belong to the crops to be extracted will also be selected, so further screening is needed. Look at Fig.5(a), the characteristic of the noise area is relatively

(C) The 2021 International Conference on Artificial Life and Robotics (ICAROB2021), January 21 to 24, 2021 
fragmentary and disconnected, so the noise can be filtered through the filtering algorithm.

Common filtering algorithms include median filtering and mean filtering. In this paper, median filtering algorithm is used to filter the image noise after testing the two effects. The effect of median filtering is shown in Fig.5(b).

\subsection{Dilate and Erode}

Dilate is the operation of finding the local maximum. In contrast to dilate, erode is the operation of finding a local minimum. Erode followed by dilate is called open operation that can remove isolated small points, while the total position and shape remain unchanged. After dilate, erode is called close operation that can fill the small holes and bridge the small gaps, which also does not change the shape and position of the image.

In this paper, we will start with the open operation to remove the noise other than the extraction of daylily. Then the close operation is used to fuse the small distance area and fill the blank area inside the image. Finally, the morphologic images are obtained.

\subsection{Choose Large Connection Domain}

After the morphological processing is completed, there are still large areas in the image that have not been filtered out, so the image can be further screened by selecting the large connection domain.

Moreover, since the mature daylily is yellow-green, there is a clear color difference from the immature daylily. The color extraction method not only separates the daylily from the branches, but also can judge whether the day-lily is mature or not. When it is mature, it can be detected and recognized, while when it is immature, it will automatically ignore the effect. The extracted daylily is shown in Fig.5(c).

\section{Experiments}

In this experiment, daylily was selected as the identified crops and were extracted from the pictures by adjusting the values of the algorithm parameters. Meanwhile, the extraction effect of tomato was also verified. But the parameter value of tomato is different from that of daylily, which also proves the universality of the algorithm.

\subsection{Daylily}

The daylily pictures used in this experiment were taken by our team members using mobile phones in the daylily planting base. The daylily is shown in Fig.4.
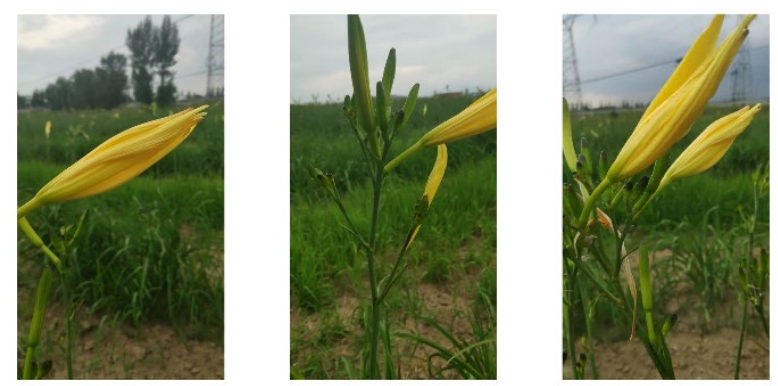

Fig.4. Daylilies original image


(a) After HSV color space extraction


(b) After morphological processing and noise filtering


(c) After selecting the large connection domain

Fig.5. Extraction results of daylilies 
The parameters used to extract daylilies are shown in Tab.1 below.

Tab.1. Parameters of daylily extraction

\begin{tabular}{ccc}
\hline Step Name & Param & Value / Range \\
\hline HSV-space & $\mathrm{H}$ & {$[0.10,0.20]$} \\
extraction & $\mathrm{S}$ & {$[0.26,1.00]$} \\
noise filter & $\mathrm{V}$ & {$[0.50,1.00]$} \\
median & - \\
$\begin{array}{c}\text { large connection } \\
\text { domain }\end{array}$ & radius & $5 \mathrm{px}$ \\
\hline
\end{tabular}

\subsection{Tomato}

At the same time, we adjusted the parameters of the algorithm to carry out the experiment of tomato extraction from Fig.6 (left), the result of which is shown in Fig.6 (right).
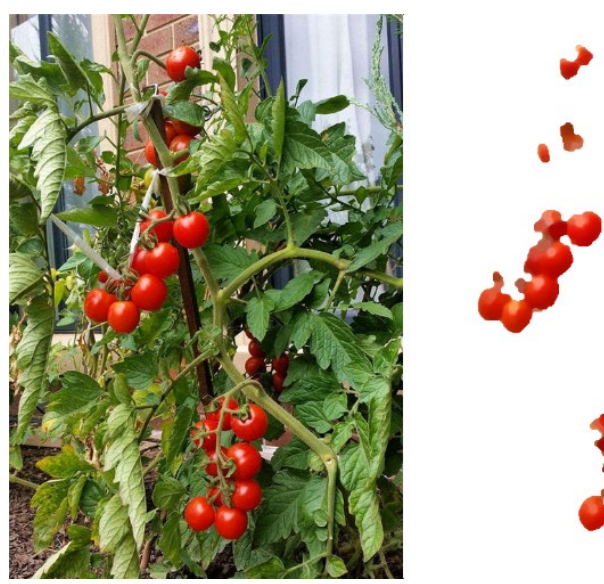

Fig.6. Tomatoes extraction

Since the extraction parameters of tomato are the same as daylily except for values of HSV, only these three items are shown in Tab.2.

Tab.2. Parameters of tomatoes extraction

\begin{tabular}{ccc}
\hline Step Name & Param & Value / Range \\
\hline HSV-space & $\mathrm{H}$ & {$[0.00,0.10]$} \\
extraction & $\mathrm{S}$ & {$[0.50,1.00]$} \\
& $\mathrm{V}$ & {$[0.50,1.00]$} \\
\hline
\end{tabular}

\section{Conclusions}

This paper presents an algorithm for crop extraction, which can extract crops with different colors before and after ripening. The algorithm was tested on daylily and tomato, and the extraction effect of the algorithm was demonstrated.

The validity and generality of the algorithm are verified by experiments. In addition, it can be seen from the experiment that this algorithm can be applied to the extraction of other crops only by adjusting the parameter values in the HSV color space.

\section{Acknowledgements}

The research is partly supported by the Project of Tianjin Enterprise Science and Technology Commissioner to Tianjin Tianke Intelligent and Manufacture Technology Co., Ltd (19JCTPJC53700). It is also supported by the Industry-University Cooperation and Education Project (201802286009) from Ministry of Education, China.

\section{References}

1. D. Khort, A. Kutyrev, et al. Robotized Platform for Picking of Strawberry Berries. 2019 IEEE International Scientific-Practical Conference Problems of Infocommunications, Science and Technology (PIC S\&T), Kyiv, Ukraine, 2019, pp. 869-872.

2. Patel, Alakh and Jaymin Bhalani. A Review on in Field Cotton Recognition for Cotton Harvesting Robot based on Image Processing Technique. International Journal of Computer Applications 180 (2018): 6-8.

3. K. N. e. Alam Siddiquee, M. S. Islam, et al. Detection, quantification and classification of ripened tomatoes: a comparative analysis of image processing and machine learning. IET Image Processing, vol. 14, no. 11, pp. 24422456, 1892020.

4. A. Wang, Y. Xu, et al. Semantic Segmentation of Crop and Weed using an Encoder-Decoder Network and Image Enhancement Method under Uncontrolled Outdoor Illumination. IEEE Access, vol. 8, pp. 81724-81734, 2020

5. M. W. Liu, M. Ozdogan and X. Zhu. Crop Type Classification by Simultaneous Use of Satellite Images of Different Resolutions. IEEE Transactions on Geoscience and Remote Sensing, vol. 52, no. 6, pp. 3637-3649, June 2014. 